University of Wollongong

Research Online

Faculty of Engineering - Papers (Archive)

Faculty of Engineering and Information

Sciences

2005

\title{
The role of nonelastic reactions in absorbed dose distributions from therapeutic proton beams in different medium
}

\author{
A. J. Wroe \\ University of Wollongong \\ I. Cornelius \\ University of Wollongong, iwan@uow.edu.au \\ Anatoly B. Rosenfeld \\ University of Wollongong, anatoly@uow.edu.au
}

Follow this and additional works at: https://ro.uow.edu.au/engpapers

Part of the Engineering Commons

https://ro.uow.edu.au/engpapers/148

\section{Recommended Citation}

Wroe, A. J.; Cornelius, I.; and Rosenfeld, Anatoly B.: The role of nonelastic reactions in absorbed dose distributions from therapeutic proton beams in different medium 2005.

https://ro.uow.edu.au/engpapers/148

Research Online is the open access institutional repository for the University of Wollongong. For further information contact the UOW Library: research-pubs@uow.edu.au 


\title{
The role of nonelastic reactions in absorbed dose distributions from therapeutic proton beams in different medium
}

\author{
Andrew J. Wroe, $\left.{ }^{a}\right)$ Iwan M. Cornelius, and Anatoly B. Rosenfeld \\ Centre for Medical Radiation Physics, University of Wollongong, Northfields Ave, \\ Wollongong, NSW, Australia, 2522
}

(Received 16 April 2004; revised 5 October 2004; accepted for publication 5 October 2004; published 14 December 2004)

\begin{abstract}
Many new techniques for delivering radiation therapy are being developed for the treatment of cancer. One of these, proton therapy, is becoming increasingly popular because of the precise way in which protons deliver dose to the tumor volume. In order to achieve this level of precision, extensive treatment planning needs to be carried out to determine the optimum beam energies, energy spread (which determines the width of the spread-out Bragg peak), and angles for each patient's treatment. Due to the level of precision required and advancements in computer technology, there is increasing interest in the use of Monte Carlo calculations for treatment planning in proton therapy. However, in order to achieve optimum simulation times, nonelastic nuclear interactions between protons and the target nucleus within the patient's internal structure are often not accounted for or are simulated using less accurate models such as analytical or ray tracing. These interactions produce high LET particles such as neutrons, alpha particles, and recoil protons, which affect the dose distribution and biological effectiveness of the beam. This situation has prompted an investigation of the importance of nonelastic products on depth dose distributions within various materials including water, A-150 tissue equivalent plastic, ICRP (International Commission on Radiological Protection) muscle, ICRP bone, and ICRP adipose. This investigation was conducted utilizing the GEANT4.5.2 Monte Carlo hadron transport toolkit. (C) 2005 American Association of Physicists in Medicine. [DOI: 10.1118/1.1824194]
\end{abstract}

Key words: proton therapy, Monte Carlo, nonelastic

\section{INTRODUCTION}

Proton therapy offers highly conformal radiotherapy treatment, which is based on specific properties of depth dose deposition by energetic protons in a medium. The energy range of protons utilized in proton therapy ranges from $60 \mathrm{MeV}$ for treatment of eye tumors up to $250 \mathrm{MeV}$ for deep-seated tumors. However, for accurate conformal treatment of the target volume it is important to have treatmentplanning systems that are able to accurately simulate the interaction of the primary proton beam within the body. Recently, a simplified Monte Carlo approach for dose planning in proton therapy for heterogeneous targets has been proposed. ${ }^{1}$ Despite advances in computer processing power, inelastic interactions are often simulated using less sophisticated analytical or ray-tracing models. ${ }^{2}$ While these models provide fast simulation times and reasonable accuracy in regions without critical heterogeneity, such models can result in errors in depth dose distributions dependent upon the positions of heterogeneous structures within the patient. ${ }^{3}$ Mistakes in the dose distribution and proton range may occur for different target materials if the proton nonelastic reactions are not considered during the absorbed dose simulations. ${ }^{4}$ Dose planning systems based on Monte Carlo simulations will be optimal for dose distributions in heterogeneous targets if the composition of the target is accurately known.

In this paper we demonstrate the importance of nonelastic proton reactions on absorbed dose distributions and proton range within homogeneous phantoms of different materials using the GEANT4.5.2 Monte Carlo simulation kit. ${ }^{5,6}$ In illustrating the importance of such interactions to treatment planning, more sophisticated methods can be employed to conduct treatment planning in heavy ion and proton therapy that will enable more accurate dose distributions to be developed. Recent developments in computer processing power, combined with modern cluster and parallel computing techniques, will minimize the cost in terms of simulation time.

\section{SIMULATION PARAMETERS OF ABSORBED DOSE IN A PROTON THERAPY BEAM}

Simulations were carried out for monoenergetic 60 and $200 \mathrm{MeV}$ proton beams within homogeneous water, A-150 tissue equivalent plastic, ICRP adipose, ICRP bone, and ICRP muscle phantoms. Each energy/tissue combination was simulated first neglecting nonelastic reactions and then considering nonelastic reactions. The depth dose distributions provided information on the importance of nonelastic reactions.

The GEANT4.5.2 Monte Carlo transport toolkit ${ }^{5}$ was used to simulate depth dose depositions within homogeneous phantoms. The phantom geometry itself consisted of a rightangled parallelepiped (RPP) volume with sensitive slices covering the cross-sectional area and extending into the phantom, Fig. 1. The sensitive slices were 5-10 $\mu \mathrm{m}$ thick separated by gaps of $0.1-1 \mathrm{~mm}$. The cross-sectional area of the sensitive slices was $5 \times 5 \mathrm{~cm}$. This provided an adequate cross-sectional area for pencil beam depth dose distributions. 


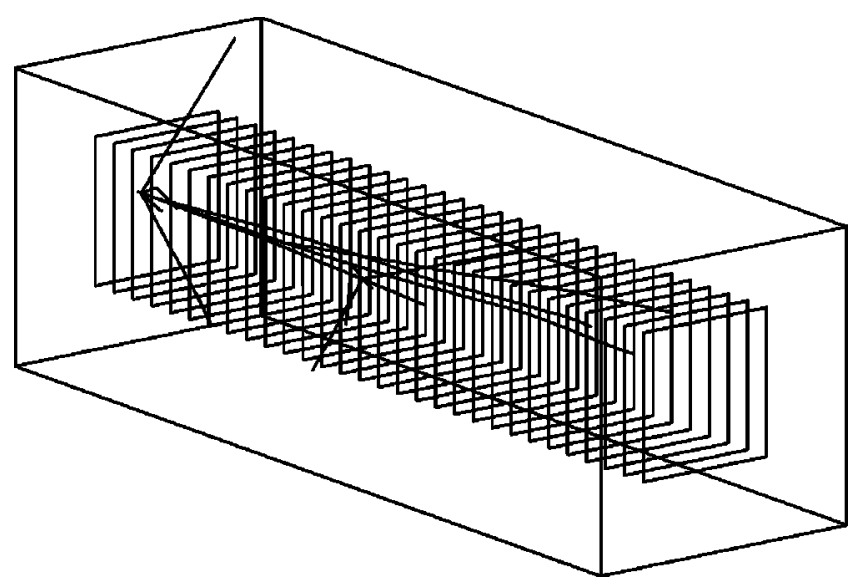

FIG. 1. A schematic representation of the phantom used in the depth dose simulations. A pencil beam of protons is traveling through the phantom and is stopping within the phantom. It is important to note the production of secondaries (photons and neutrons) as a direct result of nonelastic nuclear interactions of proton within the phantom.

In total, 320 sensitive slices were employed at regular intervals within the homogeneous phantom's structure to provide accurate resolution of the depth dose distribution. Each simulation would be run for 200000 protons that would be incident onto the phantom in the form of a pencil beam.

The physics model employed in this investigation was the G4PreCompound model. This model was deemed appropriate for this application, as it was suited to the therapeutic energy range that was to be simulated. ${ }^{6,7}$ The G4PreCompound model would account for the production of secondary particles as a result of nonelastic reactions, including the generation of charged secondaries, neutrons, and photons. This model theoretically determines nonelastic nuclear interactions and could be further verified through its comparison with doubly differential data for a number of different bombarding energies and target materials; however, this work it outside the scope of this publication. Elements making up materials utilized within the phantom geometry would be defined by isotopic abundance. This provided the most accurate composition available and was obtained from an ICRU-based program. ${ }^{8}$

To determine dose as a function of depth, the energy deposited by every particle (whether primary or secondary) within a sensitive volume was scored. This was achieved by tracking the energy depositions from both primary and secondary particles in each slice for a single incident proton history. These energy depositions were summed to provide the total energy deposited within the slice for a given history. Upon completion of the simulation, the total energy deposition in each sensitive slice was determined. From this information the absorbed dose deposited as a function of slice number or depth could be ascertained.

In order to determine the effect of nonelastic reactions on the depth dose distribution, the simulation would initially be run discounting nonelastic reactions and then again with exactly the same parameters taking into account nonelastic reactions. The depth dose distributions for both would then be compared. The comparison includes the peak to entrance dose ratio, the integral dose, the Bragg peak position, and entrance dose.

\section{SIMULATION VERIFICATION}

To verify the Monte Carlo simulation, GEANT4 code simulations of absorbed dose for a $160.7 \mathrm{MeV}$ proton beam of Guassian width $\sigma=0.8035 \mathrm{MeV}$ within a homogeneous water phantom were compared with GEANT4 simulation data supplied by Paganetti from the NPTC (Northeast Proton Therapy Center), MGH (Massachusetts General Hospital), Boston. The simulation data supplied by Paganetti utilized the same incident beam properties, and the phantom consisted of a homogeneous water tank with a $10 \mathrm{~mm}$ thick Perspex lid. The GEANT4 simulation of Paganetti has undergone extensive experimental verification at NEPC and can be considered a good benchmark for the GEANT4.5.2 utilized in this research. Figure 2 shows simulated and experimentally verified simulation data.

There was excellent agreement between the two data series for Bragg peak position, FWHM, plateau dose, and distal edge. There was a slight shift in the Bragg peak and no verified simulation data were provided in the first $10 \mathrm{~mm}$ of the distribution due to a $10 \mathrm{~mm}$ thick Perspex lid situated at the surface of the water tank. The small shift in the Bragg peak can be accounted for using the linear stopping power of protons within the two materials.

A proton traversing the two materials will expend approximately $1 \mathrm{MeV}$ of energy more traveling through $1 \mathrm{~cm}$ of Perspex as opposed to through $1 \mathrm{~cm}$ of water. Using the linear stopping powers from the above table, it is possible to determine that for an incident proton energy of $160.7 \mathrm{MeV}$, after traversing $1 \mathrm{~cm}$ of Perspex the proton will have an energy of $154.7 \mathrm{MeV}$, and after traversing $1 \mathrm{~cm}$ of water the proton will have an energy of $155.5 \mathrm{MeV}$. The difference in range can be calculated using data sourced from the ICRU Projected Range Data for water. ${ }^{4}$ When this is considered,

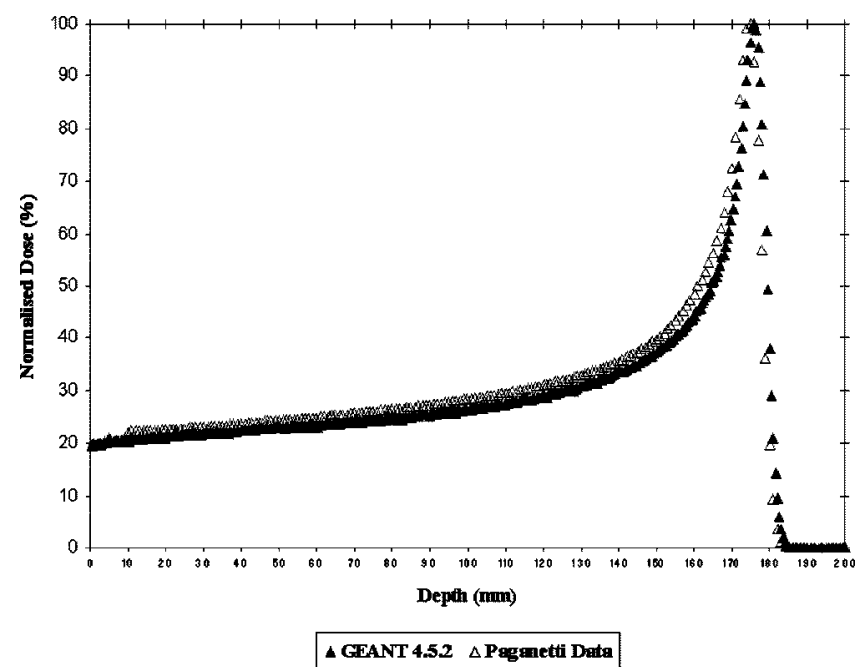

FIG. 2. Comparison of simulation data supplied by Paganetti and GEANT4.5.2 simulation utilized in this investigation for a $160.7 \mathrm{MeV}$ proton beam incident on a water phantom. Data were normalized to the peak dose. 
TABLE I. Material data for Perspex and water obtained for a $160.7 \mathrm{MeV}$ proton beam (Ref. 4).

\begin{tabular}{cccc}
\hline \hline Material & $\begin{array}{c}\text { Density of material } \\
\left(\mathrm{g} / \mathrm{cm}^{3}\right)\end{array}$ & $\begin{array}{c}\text { Total stopping power } \\
\left(\mathrm{MeV} \mathrm{cm}^{2} / \mathrm{g}\right)\end{array}$ & $\begin{array}{c}\text { Linear stopping } \\
\text { power }(\mathrm{MeV} / \mathrm{cm})\end{array}$ \\
\hline Water & 1.000 & 5.193 & 5.193 \\
Perspex & 1.19 & 5.055 & 6.016 \\
\hline \hline
\end{tabular}

the difference in range is $0.15 \mathrm{~cm}$ and accounts for the difference in Bragg peak position between the two simulations. Similar simulations were conducted for 60, 200, and $250 \mathrm{MeV}$ incident proton energies from three other sources, and in all cases the GEANT4.5.2 simulation compared extremely well. As such, it can be concluded from this analysis that our Monte Carlo simulation with the GEANT4.5.2 is in agreement with the experimentally confirmed work of Paganetti.

\section{RESULTS}

Simulations were carried out for monoenergetic 60 and $200 \mathrm{MeV}$ proton beams within homogeneous water, A-150 tissue equivalent plastic, ICRP adipose, ICRP bone, and ICRP muscle phantoms. Each energy/tissue combination was simulated for 200000 incident protons first neglecting nonelastic reactions and then considering them. This was achieved within GEANT by inactivating and then activating the nonelastic interaction parameter. The depth dose distributions for these provided information on the importance of nonelastic reactions. Figures 3 and 4 show the importance of nonelastic reactions on dose distribution in different phantom materials for monoenergetic 60 and $200 \mathrm{MeV}$ proton beams, respectively.

The results for both proton energies in Figs. 3 and 4 clearly demonstrate the effect of composition of the phantom

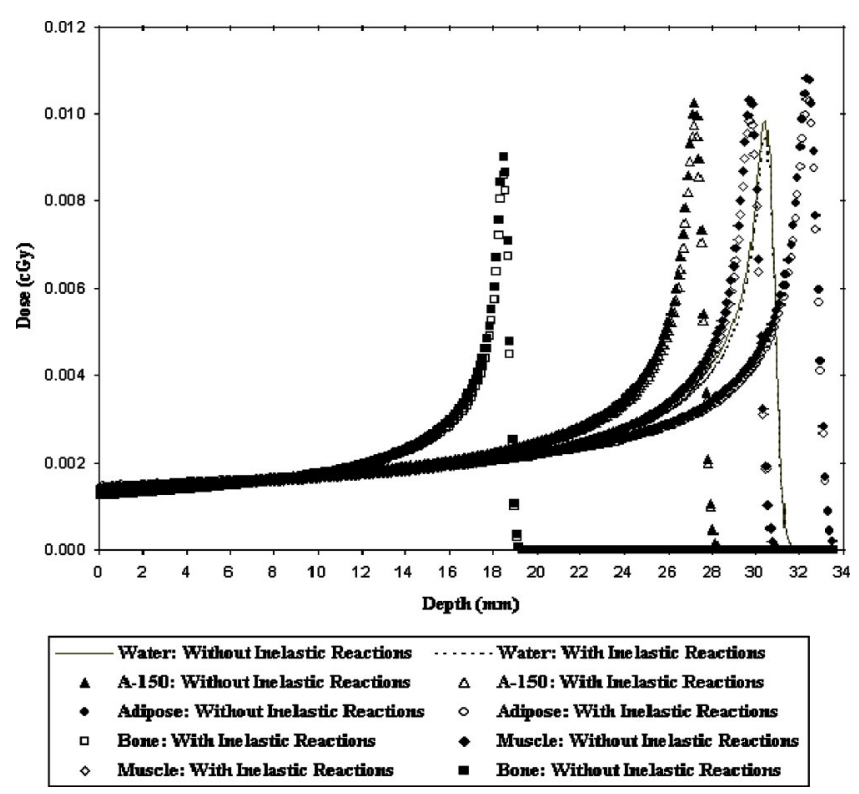

FIG. 3. Comparison of depth dose distributions for a monoenergetic $60 \mathrm{MeV}$ proton beam incident on different phantom materials.
TABLE II. The Bragg peak position, ratio of peak to entrance dose, and the difference in this ratio between the simulation that accounts for nonelastic reactions and the one that does not for the $60 \mathrm{MeV}$ depth dose distribution.

\begin{tabular}{ccccc}
\hline \hline & $\begin{array}{c}\text { Bragg peak } \\
\text { position } \\
(\mathrm{mm})\end{array}$ & $\begin{array}{c}\text { Ratio of peak to } \\
\text { entrance dose } \\
\text { without nonelastic } \\
\text { reactions }\end{array}$ & $\begin{array}{c}\text { Ratio of peak to } \\
\text { entrance dose } \\
\text { with nonelastic } \\
\text { reactions }\end{array}$ & $\begin{array}{c}\text { Difference } \\
\text { in ratio } \\
(\%)\end{array}$ \\
\hline Water & 30.45 & 7.017 & 6.694 & 4.82 \\
A-150 & 27.20 & 7.353 & 6.822 & 7.78 \\
Adipose & 32.44 & 7.569 & 7.136 & 6.07 \\
Bone & 18.45 & 7.140 & 6.729 & 6.11 \\
Muscle & 29.82 & 7.478 & 7.051 & 6.06 \\
\hline \hline
\end{tabular}

on Bragg peak position. Additionally, the role of nonelastic reactions can be seen in amplitude (shape) change of the Bragg peak when nonelastic interactions have been taken into account in absorbed dose simulations. Figure 4 shows that this difference in amplitude becomes much more pronounced with increasing proton energy.

It is clear from the 60 and $200 \mathrm{MeV}$ results that there is indeed an effect not only on the range of the protons within the phantom but also on the nonelastic reactions component as a result of phantom composition. In the case of $60 \mathrm{MeV}$ protons the difference in Bragg peak position was approximately $14 \mathrm{~mm}$ across the simulated materials; however, in the case of the $200 \mathrm{MeV}$ proton beam this effect was almost $120 \mathrm{~mm}$. As expected, the stopping power predominantly determined the range of the primary protons within different materials. This parameter is already utilized in many treatment-planning systems to determine any differences in range that is encountered as a result of a heterogeneous patient geometry. The importance of accurate geometry composition is apparent, as variations in composition can result in a large difference between the position of the simulated Bragg peak position and the actual position within the phantom or patient. This error in Bragg peak position could lead to excessive dose being delivered to sensitive structures located near the tumor volume.

The simulated position of the Bragg peak within the phantom did not alter between that which did and did not account for nonelastic reactions in water, muscle, adipose, A-150, and bone. This was shown to be the case for both proton energies simulated in this study.

Clearly, nonelastic reactions affect the amplitude of the Bragg peak as dose is deposited either outside the sensitive slices or at different regions of the proton's track. To illustrate this more clearly, the peak to entrance dose ratios for both simulations are contained within Tables II and III. The peak to entrance dose ratio quantifies a decrease in the amplitude of the Bragg peak and also any increase in the entrance dose. The difference in the peak to entrance dose ratio is also contained within Tables II and III, and is expressed as a percentage.

The data in Tables II and III show that as the incident proton energy increased the cumulative effect of nonelastic reactions also increased. A decrease in peak to entrance dose 


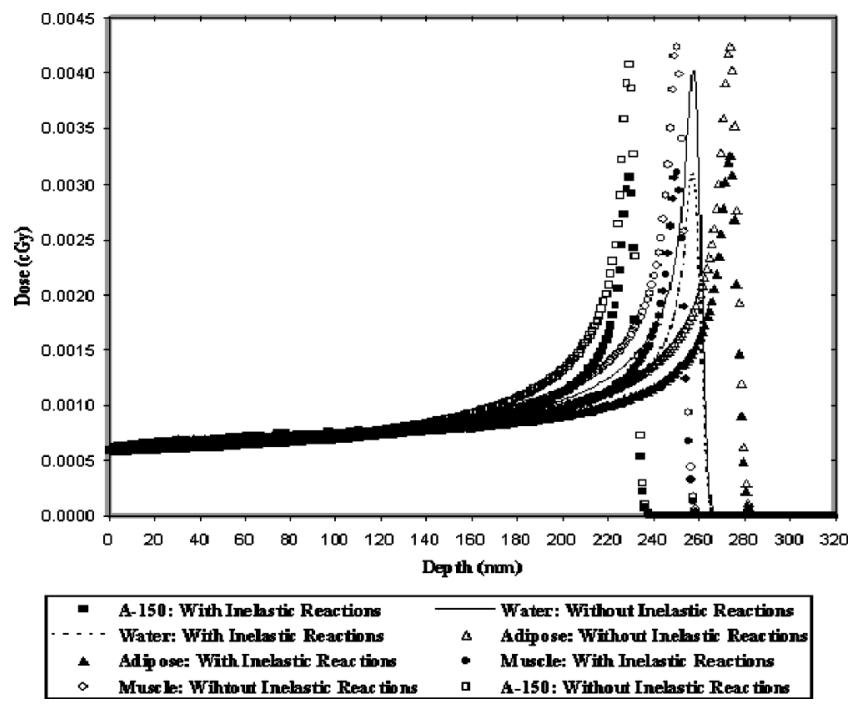

FIG. 4. Comparison of depth dose distributions for a monoenergetic $200 \mathrm{MeV}$ proton beam in different phantom materials.

ratio was observed to be around a $5 \%-8 \%$ for $60 \mathrm{MeV}$ protons and over $30 \%$ for the $200 \mathrm{MeV}$ beam. As the nonelastic cross section depends on proton energy and target composition, these effects can be dominated by different materials for different proton energies. A-150 was the most affected material at $60 \mathrm{MeV}$. However, at $200 \mathrm{MeV}$ the most affected material was muscle. These effects are a direct result of different elemental compositions of the different materials producing different nonelastic cross sections at different energies.

The decrease in Bragg peak amplitude is a clear indication of the effect that nonelastic reactions have on the depth dose distribution. Failure to account for this accurately could result in significant dose errors in the Bragg peak region. However, reduction in Bragg peak amplitude is not the only effect that resulted from the inclusion of nonelastic reactions in the simulation model. There was also a small increase in the entrance dose region, as well as an increase in the dose deposited past the Bragg peak as a result of secondary particles produced by nonelastic nuclear reactions. However, these are not clearly visible on the depth dose distributions

TABLE III. The Bragg peak position, ratio of peak to entrance dose, and the difference in this ratio between the simulation that accounts for nonelastic reactions and the one that does not for $200 \mathrm{MeV}$ depth dose distribution.

\begin{tabular}{lcccc}
\hline \hline & $\begin{array}{c}\text { Ratio of peak to } \\
\text { entrance dose } \\
\text { without nonelastic } \\
\text { reactions }\end{array}$ & $\begin{array}{c}\text { Ratio of peak to } \\
\text { entrance dose } \\
\text { with nonelastic } \\
\text { reactions }\end{array}$ & $\begin{array}{c}\text { Difference } \\
\text { in ratio } \\
(\%)\end{array}$ \\
\hline Water & 257.55 & 6.872 & 5.134 & 33.8 \\
A-150 & 229.27 & 6.999 & 5.106 & 37.1 \\
Adipose & 273.71 & 7.042 & 5.331 & 32.0 \\
Muscle & 250.48 & 7.128 & 5.141 & 38.6 \\
\hline \hline
\end{tabular}

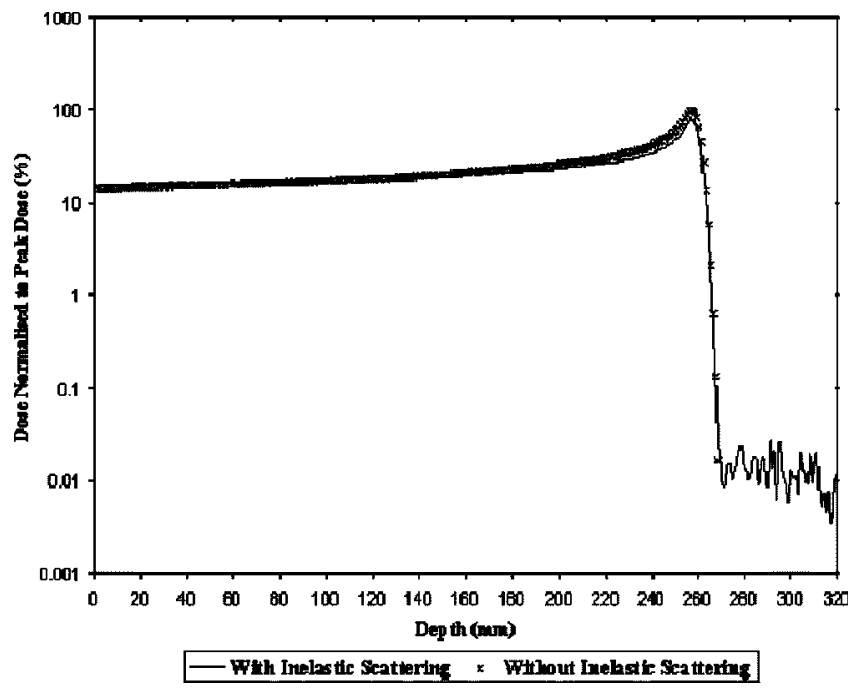

FIG. 5. $200 \mathrm{MeV}$ pencil proton beam depth dose distribution in a water phantom plotted on a logarithmic graph. The dose in this distribution has been normalized to the peak dose.

supplied in Figs. 3 and 4. A depth dose distribution of both simulations plotted on a logarithmic scale is required to observe these effects, as shown in Fig 5.

It is clear from Fig. 5 that in the case of the simulation that includes nonelastic reactions some dose is deposited past the Bragg peak as a result of long-range nonelastic products such as neutrons and photons. This dose is orders of magnitude smaller than the peak dose; however, it may be important to consider. For example, in proton therapy it is routine to superimpose a number of Bragg peaks in order to achieve a dose distribution of adequate width to treat the tumor volume. In doing so, the tail from each Bragg peak will also be superimposed, increasing dose past the tumor volume. This can be considered even more significant as this tail is made up of neutrons that may increase the biological dose in this region. This dose could be significant for sensitive structures located close to the distal edge of the SOBP (spread-out Bragg peak).

The phantom material also affects the spectra of secondary particles produced via nonelastic reactions. This is clear from Table IV when we consider the difference in integral dose between the simulation that accounts for nonelastic reactions and the one that does not for the $200 \mathrm{MeV}$ incident

TABLE IV. The integral dose and the difference in integral dose between the simulation that accounts for nonelastic reactions and the one that does not for $200 \mathrm{MeV}$ depth dose distribution. Integral dose in cGy per 200000 protons.

\begin{tabular}{cccc}
\hline \hline Material & $\begin{array}{c}\text { Integral dose } \\
\text { without nonelastic } \\
\text { reactions }\end{array}$ & $\begin{array}{c}\text { Integral dose } \\
\text { with nonelastic } \\
\text { reactions }\end{array}$ & Difference (\%) \\
\hline Water & 0.25 & 0.24 & 7.7 \\
A-150 & 0.23 & 0.21 & 7.9 \\
Adipose & 0.28 & 0.26 & 8.0 \\
Muscle & 0.26 & 0.23 & 12.0 \\
\hline \hline
\end{tabular}


proton beam. Of all the materials, simulated muscle has the highest variation between the simulation that considers nonelastic reactions and the one that does not. This is possibly a direct result of muscle producing more long-range secondaries such as neutrons and photons, which carry dose out of the sensitive slices and away from the incident proton's track. This would be a direct result of the composition of the material and the different nonelastic cross sections of the different elements. It is also important to note that the pencil beam of protons was incident on the center of a $50 \times 50 \mathrm{~mm}$ sensitive slice, and as such in order to deposit dose outside the sensitive slice, the secondary particles had to be significantly deviated from the incident proton's track. The difference in the integral dose would have increased significantly if the cross-sectional area of the sensitive slices had been reduced for example to $1 \mathrm{~cm}^{2}$, as this would have resulted in a greater fraction of the dose being deposited away from the proton's track outside the sensitive slices.

\section{CONCLUSION}

Inelastic secondary products are shown to be an important consideration to the depth dose distribution of therapeutic proton beams. Different phantom materials produce different levels of nonelastic reactions and different nonelastic products. The spectra of secondary particles and the cross section for nonelastic reactions also changes as a function of energy. Thus, accurate patient geometry and composition needs to be accurately included in the treatment-planning system. Secondary particles also need to be tracked and affect the interaction scored. This can be achieved using Monte Carlo based treatment planning systems.

The results presented within this article demonstrate that nonelastic reactions do not affect the position of the Bragg peak within the phantom. The current study highlighted that nonelastic reactions affect the amplitude of the Bragg peak with reductions of nearly $40 \%$ in the peak to entrance dose ratio. The effect of nonelastic reactions was shown to increase with increasing incident proton energy, and was also seen to be dependent upon the phantom material. These results highlight the need for accurate material compositions and cross sections of nonelastic reactions within treatmentplanning systems, as the cross sections of nonelastic reactions and the spectra of secondary particles appeared to vary between materials and with incident proton energy. It could be possible to implement this into a dose planning system that assigns for each voxel a composition that can be obtained from CT numbers and MRI, using identification of the organ and established composition data tables for particular organs and structures. These tables could be built into the system to associate composition with CT number and hence assign elemental composition to each voxel within the CT image. It is important to mention that the uncertainty in nonelastic cross sections will affect dose distributions within a phantom.

The simulations also showed that nonelastic secondary particles increased the dose in the tail of the Bragg peak. The dose in the tail is of the order of three decades smaller than the peak dose. However, the superposition of a number of peaks in order to form the spread-out Bragg peak (SOBP) will result in the superposition of this tail. This could result in a biologically significant dose to sensitive structures, especially if they are located close to the treatment volume.

\section{ACKNOWLEDGMENTS}

Thanks to Dr. H. Paganetti, NEPC, for providing data on proton absorbed dose in a water phantom. Also, thanks needs to be extended to Dr. R. Schulte, Loma Linda Medical Center, for useful discussions during this work. This work was completed with the support of the Australian Institute of Nuclear Science and Engineering and the Australian Proton Therapy Group.

\footnotetext{
${ }^{a)}$ Electronic mail: ajw16@uow.edu.au

${ }^{1}$ R. Kohno, Y. Takada, T. Sakae, T. Terunuma, K. Matsumoto, A. Nohtomi, and H. Matsuda, "Experimental evaluation of validity of simplified Monte Carlo method in proton dose calculations," Phys. Med. Biol. 48, 12771288 (2003).

${ }^{2}$ L. Petti Paula, "Differential-pencil-beam dose calculations for charged particles," Med. Phys. 19, 137-149 (1992).

${ }^{3}$ B. Schnaffner, E. Pedroni, and A. Lomax, "Dose calculation models for proton treatment planning using a dynamic beam delivery system: An attempt to include density heterogeneity effects in the analytical dose calculation," Phys. Med. Biol. 44, 27-41 (1999).

${ }^{4} \mathrm{H}$. Paganetti, "Nuclear interactions in proton therapy: Dose and relative biological effect distributions originating from primary and secondary particles," Phys. Med. Biol. 47, 747-764 (2002).

5 GEANT4 User's Guide-For Application Developers, GEANT4 User's Documents, December 2002. http://wwwasd.web.cern.ch/wwwasd/ geant4/G4UsersDocuments/UsersGuides/ForApplicationDeveloper/html/ index.html

${ }^{6}$ GEANT4 Physics Reference Manual, GEANT4 User's Documents, January 2003. http://wwwasd.web.cern.ch/wwwasd/geant4/G4UsersDocuments/ UsersGuides/PhysicsReferenceManual/html/PhysicsReferenceManual .html

${ }^{7}$ I. Cornelius, A. Rosenfeld, and P. Bradley, "Simulations of microdosimetry measurements in fast neutron therapy," Australas. Phys. Eng. Sci. Med. 25, 168-171 (2002).

${ }^{8}$ National Institute of Standards and Technology (NIST) PSTAR Database Program. http://physics.nist.gov/cgi-bin/Star/compos.pl?ap.
} 